\title{
Desafios das Fake News com Idosos durante Infodemia sobre Covid-19: Experiência de Estudantes de Medicina
}

\author{
Challenges Caused by Fake News among Elderly Population during the Covid-19 Infodemic: Experience of \\ Medical Students
}

\author{
Angela Theresa Zuffo Yabrude ${ }^{1(0)}$, Andressa Caroline Martins de Souza"(®), \\ Catarine Wiggers de Campos ${ }^{\prime \prime \prime}$, Loyse Bohn"(1), Marcela Tiboni'(D)
}

\begin{abstract}
RESUMO
Introdução: A pandemia do novo coronavírus trouxe consigo uma infodemia, ou seja, um excesso de informações, que, em populações com baixa análise crítica e falta de conhecimento técnico-científico, pode gerar e disseminar fake news. No Brasil, esses déficits são encontrados frequentemente nos idosos, que representam 13\% da população, e na maior parte dos analfabetos absolutos e funcionais, o que os torna tanto vítimas quanto propagadores.

Relato de Experiência: Foi realizada uma atividade multicêntrica baseada no projeto-piloto da Faculdade Evangélica Mackenzie do Paraná que promoveu educação em saúde para a população idosa por meio de redes sociais e comunicação on-line. Assim, os estudantes de Medicina ficaram disponíveis para esclarecer dúvidas e mitos relacionados à Covid-19 e enviar materiais informativos. Doze instituições de ensino superior da Região Sul do Brasil replicaram o projeto de 4 de julho a 6 de agosto de 2020, com o objetivo de combater às fake news e estimular a criação de canais de comunicação confiáveis com essa população.

Discussão: A inclusão digital do idoso é algo recente, e a proporção daqueles que são usuários da internet vem crescendo no país. Entretanto, ainda há baixa interpretação crítica de informações, dificuldade de acompanhar o fluxo de notícias e pouca habilidade com ferramentas da internet. Nesse sentido, dar protagonismo a essa população digitalmente invisibilizada e permitir a ampliação do conhecimento médico geriátrico durante a pandemia, por meio do contato de acadêmicos com as demandas dos idosos, é uma forma efetiva de possibilitar um entendimento maior acerca das vulnerabilidades e necessidades do público geriátrico no que tange à educação em saúde.
\end{abstract}

Conclusão: A construção do canal de comunicação entre acadêmicos e idosos apresentou uma possibilidade inovadora para a população idosa obter informação científica de forma acessível, de modo a conscientizá-la do novo coronavírus e da propagação de notícias falsas.

Palavras-chave: Inclusão Digital; Geriatria; Acesso à Informação; Populações Vulneráveis; Coronavírus

\begin{abstract}
Introduction: The Coronavirus pandemic brought with it an infodemic, which is an excess of information that, in environments with low critical analysis and a lack of technical-scientific knowledge, generates propitious ground for the emergence and dissemination of fake news. In Brazil, these weaknesses are widely found among the elderly, which represents $13 \%$ of the population and the majority of absolute and functional illiterates, making them both victims and propagators.

Experience Report: A multicenter activity was carried out based on the pilot project by Faculdade Evangélica Mackenzie do Paraná, which promoted health education for the elderly population through social networks and online communication. Thus, medical students made themselves available to clarify their doubts and other myths related to COVID-19 and share informational materials. Twelve medical schools in the southern region of Brazil replicated the project from 4th July to 6 th August 2020, with the aim of combating fake news and encouraging the creation of communication channels with this population.

Discussion: Digital inclusion of the elderly is recent and the proportion of elderly people who are Internet users has been growing in the country. However, there is still a low critical interpretation of information, difficulty in keeping up with the news flow and lack of skill with Internet tools. In this sense, giving prominence to this digitally invisible population and allowing the expansion of geriatric medical knowledge during the pandemic by increasing students' contact with the demands of the elderly, is an effective way of enabling a greater understanding about the vulnerabilities and needs of the elderly regarding health education.
\end{abstract}

Conclusion: The construction of a communication channel between students and the elderly is an innovative possibility for this group to enjoy easy access to scientific information, raising awareness concerning the coronavirus and fake news.

Keywords: Digital Inclusion; Geriatrics; Access to Information; Vulnerable Populations; Coronavirus.

'Universidade Regional de Blumenau, Blumenau, Santa Catarina, Brasil.

"Faculdade Evangélica Mackenzie do Paraná, Curitiba, Paraná, Brasil.

"'Universidade do Sul de Santa Catarina, Tubarão, Santa Catarina, Brasil

Correspondência:

Marcela Tiboni.

Rua Alfredo Gunther, 42, ap. 23, Itoupava Seca, Blumenau, SC, Brasil. CEP: 89030-108.

E-mail:ma.tiboni@gmail.com

Recebido em 11/08/20; Aceito em 28/08/20. 


\section{INTRODUÇÃO}

Vivendo-se em um mundo altamente globalizado, com o crescente uso das redes sociais e o amplo acesso a elas, as informações tendem a se espalhar rapidamente, o que muitas vezes ocorre sem que haja a correta aferição de sua veracidade ${ }^{1,2}$. Com a pandemia da coronavirus disease 2019 (Covid-19) sendo declarada mundialmente em março de 2020 pela Organização Mundial da Saúde (OMS), essa conjuntura amplificou-se e gerou o fenômeno caracterizado como infodemia - um excesso de informações que dificulta a veiculação de notícias de fontes confiáveis ${ }^{3,4}$.

A infodemia impõe risco ao acesso às fontes confiáveis por parte da população, visto que nas redes sociais as informações com cunho pessoal ou notícias intencionalmente falsas são espalhadas rapidamente e por usuários mais influentes, em detrimento do compartilhamento de dados científicos que acabam não tendo o mesmo alcance ${ }^{2}$. Durante a pandemia da Covid-19, no Reino Unido, foi descrito que $46 \%$ dos adultos referiram exposição a informações não verdadeiras na internet e que $40 \%$ consideram difícil distinguir as notícias falsas das verdadeiras 5 . Esse fenômeno é conjuntamente crescente no contexto da pandemia no Brasil, somado à falta de conhecimento técnico-científico e pensamento crítico, que se explicita no fato de mais de 11,5 milhões de brasileiros acima de 15 anos serem analfabetos absolutos e 38 milhões de pessoas serem analfabetas funcionais ${ }^{6}$.

Como se trata de um país que se projeta com mais de 28 milhões de pessoas com mais de 60 anos de idade, há uma preocupação pertinente com a população idosa, pois a presença dos idosos como usuários da internet vem crescendo e se mostrou com o maior aumento proporcional entre as faixas etárias. Além disso, são caracterizados como população de risco para a Covid-19, possuindo altos índices de hospitalização, taxa de complicações e elevada letalidade ${ }^{7-9}$. Dessa maneira, a divulgação de notícias intencionalmente falsas acabou por se tornar algo cotidiano para o grupo geriátrico, e, à medida que o compartilhamento dessas informações, mesmo não verídicas, influencia a opinião pública em geral, isso possibilita o abandono de tratamentos, suscita interações medicamentosas e pode agravar doenças preexistentes, colocando em risco a saúde dos próprios idosos e de seus familiares ${ }^{10,11}$. Como o combate às fake news é mais um desafio para literacia em saúde, há uma discussão sobre o desenvolvimento de estratégias formativas no ensino superior para que estudantes e professores reconheçam seu papel de intervenção ${ }^{12}$.

Nesse contexto, foi desenvolvida uma ação por acadêmicos de Medicina cujo propósito é fornecer aos idosos notícias e artigos de fontes confiáveis, por meio do aplicativo WhatsApp, para esclarecer dúvidas e minimizar o compartilhamento de informações falsas por essa população.

\section{RELATO DE EXPERIÊNCIA}

O presente estudo se caracteriza como observacional, descritivo e retrospectivo que retrata a experiência de quatro acadêmicas de Medicina na coordenação de um projeto multicêntrico de promoção da saúde pública. As ações visaram combater a "infodemia" durante a pandemia da Covid-19 e promover a educação em saúde entre as populações vulneráveis, tendo como cerne da proposta os idosos. O objetivo foi divulgar informações fidedignas e sanar dúvidas sobre a Covid-19, além de manter um contato ativo entre o acadêmico de Medicina e essa população, com base na necessidade curricular de ensino-aprendizagem sobre gerontologia. Um resumo de como ocorreu a estruturação dos trabalhos pode ser visto na Figura 1.

Primeira e segunda fases: Projeto-piloto, elaboração e divulgação da atividade multicêntrica

O projeto-piloto ocorreu na Faculdade Evangélica Mackenzie do Paraná (Fempar) em maio de 2020, e, em razão dos resultados e da abrangência dele, foi proposta a realização da atividade em outras cidades da Região Sul do Brasil. As universidades foram selecionadas de acordo com um formulário de inscrição, disponibilizado na lista de e-mail de uma instituição de representatividade estudantil de âmbito nacional. Das instituições de ensino superior inscritas, 12 replicaram o projeto: Fempar, Universidade Regional de Blumenau, Universidade Federal de Pelotas, Pontifícia Universidade Católica do Paraná, Universidade Positivo, Universidade de Caxias do Sul, Universidade de Santa Cruz do Sul, Universidade do Oeste de Santa Catarina, Universidade da Região de Joinville, Faculdade Campo Real, Universidade do Oeste do Paraná (campus de Francisco Beltrão) e Universidade Comunitária da Região de Chapecó. Cada escola médica contou com um grupo de acadêmicos que ficou responsável pela organização interna e pelo feedback às coordenadoras da atividade multicêntrica, a fim de propiciar a realização das atividades, as quais ocorreram simultaneamente de 4 de julho 6 de agosto de 2020. Houve ainda a participação de acadêmicos voluntários.

Terceira fase: Estruturação da atividade multicêntrica com as escolas médicas participantes

Iniciou-se a atividade multicêntrica com uma capacitação proposta pelas estudantes coordenadoras voltada aos acadêmicos organizadores de cada escola médica acerca do projeto-piloto da Fempar. Dessa forma, foi possível 
Figura 1. Fluxograma esquemático da metodologia da atividade multicêntrica

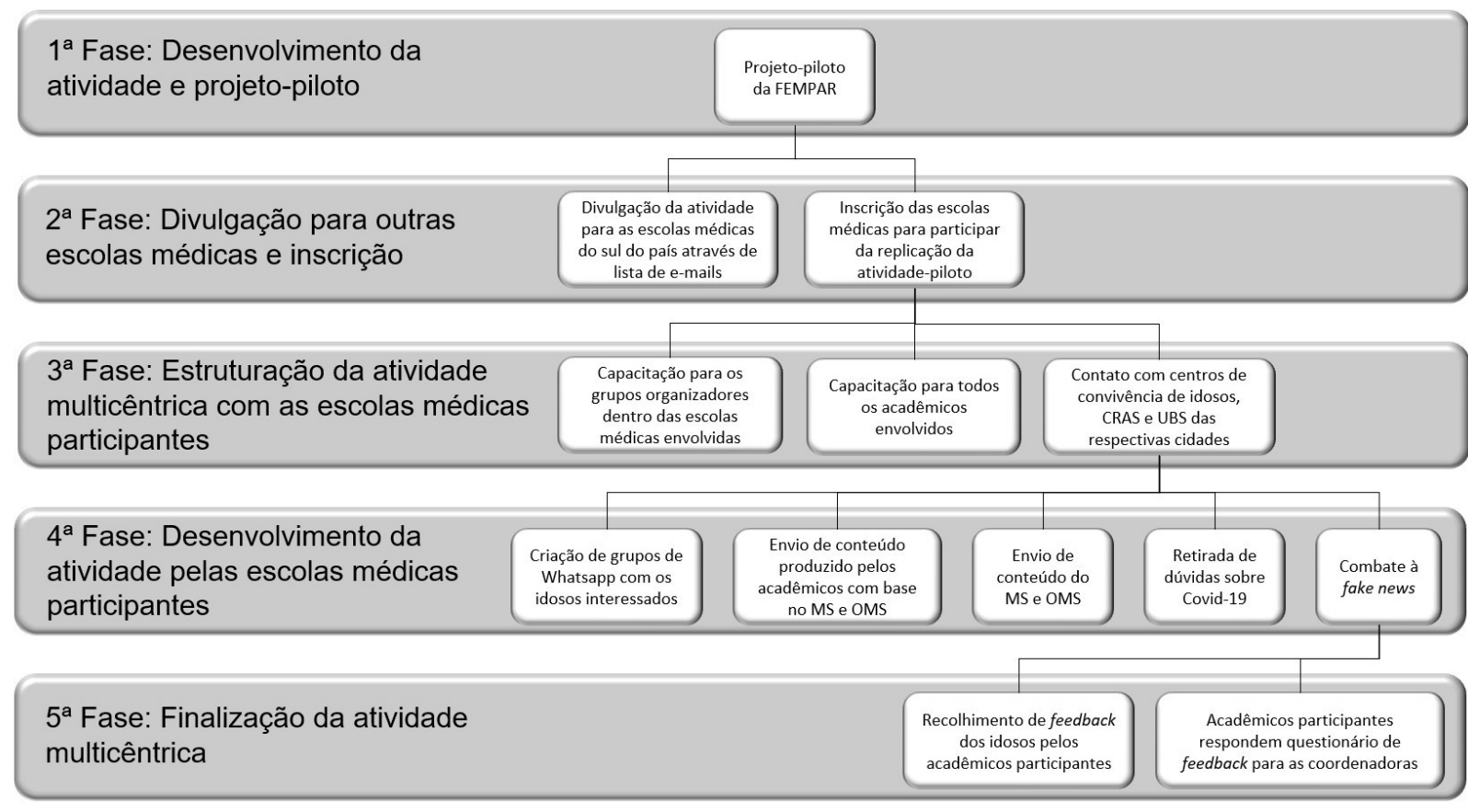

Fonte: Elaborada pelas autoras.

dialogar sobre metas, propósitos e metodologias a serem utilizadas para a população selecionada. Houve uma segunda capacitação organizada pelas coordenadoras para todos os participantes, organizadores e voluntários, que aconteceu em dois momentos:

- No primeiro, os acadêmicos foram capacitados por uma infectologista por meio de uma aula que abordava os conhecimentos atuais sobre a Covid-19, as fake news mais comuns e os modos de refutá-las.

- No segundo, uma fisioterapeuta especialista em gerontologia conduziu uma aula sobre a melhor forma de se comunicar com o público-alvo e sobre os métodos a serem utilizados pelos idosos a fim de que possam se manter ativos e saudáveis durante o isolamento social.

As coordenadoras da atividade multicêntrica disponibilizaram previamente aos participantes material que continha as perguntas realizadas mais frequentemente pelos idosos na atividade-piloto, além de informações recentes da OMS e do Ministério da Saúde (MS) sobre o novo coronavírus.

Após as capacitações, os acadêmicos contataram grupos de unidades básicas de saúde (UBS), centros de referência de assistência social (Cras), lares de longa permanência para idosos e programas de extensão voltados à saúde do idoso com a finalidade de fazer uma intermediação com essa população nos municípios. Posteriormente, os grupos de acadêmicos (organizadores e voluntários) de cada instituição de ensino participante criaram um grupo no aplicativo WhatsApp com os idosos que consentiram com a participação, para estabelecer esse canal de comunicação e compartilhamento de informações. No total, a ação contou com 119 acadêmicos envolvidos e 375 idosos impactados. Durante toda a atividade multicêntrica, houve feedback contínuo às coordenadoras por parte dos acadêmicos organizadores.

Quarta fase: Desenvolvimento da atividade multicêntrica pelas escolas médicas participantes

Nos grupos entre os acadêmicos voluntários e idosos, houve compartilhamento de conteúdo e materiais de confecção própria, com base nas referências da OMS e do MS, ou mesmo materiais informativos previamente produzidos por essas instituições com as seguintes temáticas: pandemia do novo coronavírus e os sintomas; uso de máscaras; como entrar em contato com o teleatendimento local ou nacional; saúde e bem-estar durante tempos de isolamento social; e como identificar fake news.

Além disso, os idosos poderiam fazer perguntas e tirar dúvidas conforme demanda, e, para tanto, foram orientados a enviá-las quando sentissem necessidade. Os questionamentos foram respondidos pelos discentes por meio de áudios e vídeos, nos quais estes se identificavam e faziam os devidos esclarecimentos. Ademais, como novas informações sobre a Covid-19 poderiam surgir a qualquer momento, indicou-se a necessidade de sempre mencionar a data de envio do áudio. Para responder às questões, os estudantes contaram com a 
ajuda de alguns professores e se basearam nas informações do MS e da OMS. As perguntas mais frequentes foram: "Quais são os principais sintomas?", "Estou [ou algum familiar] está com sintomas, o que deve ser feito?", "Como higienizo meus pertences?" e "Como me proteger do novo coronavírus?". $\mathrm{Na}$ maioria das vezes, as questões versavam sobre transmissão, prevenção, sintomatologia e tratamento.

No decorrer do projeto, os acadêmicos organizadores e voluntários conseguiram manter um bom fluxo de comunicação com os idosos, propiciando um diálogo sobre o assunto em questão.

\section{Quinta fase: Finalização da atividade multicêntrica}

As coordenadoras incentivaram os acadêmicos a obter feedback dos idosos sobre o impacto da ação, o que aconteceu, inicialmente, por meio de formulários on-line. Houve limitação quanto ao uso de websites de formulários que requeriam troca de aplicativo para avaliação do impacto e feedback da ação pelos idosos. Perceberam-se adversidades relacionadas ao entendimento sobre como acessar o formulário, problemas de compreensão das questões propostas, exaustão em responder a elas ou falta de interesse. No entanto, essas limitações foram sanadas pelo incentivo ao feedback por meio de relatos feitos no WhatsApp. Os relatos foram, em unanimidade, positivos com relação à ação dos estudantes de Medicina.

Outra limitação encontrada se caracteriza por dificuldades em encontrar grupos de idosos dispostos a participar da atividade.

\section{DISCUSSÃO}

No contexto da pandemia, como há uma sobrecarga de informações, torna-se difícil controlar a qualidade e autenticidade delas, o que pode gerar o aumento no número de fake news. Esse processo, caraterizado como infodemia, foi reconhecido por agravar o contexto da pandemia da Covid-19, manifestando-se como um ciclo perigoso que pode acelerar e propagar a desinformação ${ }^{13}$. Existem diversas explicações para o amplo compartilhamento de notícias falsas, e as principais incluem falta de conhecimento técnico-científico e baixa análise crítica sobre a veracidade de um conteúdo'.

Nesse universo, a população idosa merece destaque por ser mais vulnerável à propagação das fake news, pois os indivíduos acima de 65 anos de idade são sete vezes mais propensos a espalhar notícias falsas do que as pessoas com menos de 29 anos. As hipóteses para essa suscetibilidade incluem, além do posicionamento político-comportamental, o analfabetismo absoluto e o funcional presentes na população idosa ${ }^{14}$. No Brasil, a taxa de analfabetismo no grupo populacional de 60 anos ou mais chega a 10,3\% entre brancos e amplia-se para $27,5 \%$ em pretos e pardos, além de $53 \%$ das pessoas entre 50 e 64 anos serem consideradas analfabetas funcionais. Assim, o precário domínio das habilidades de leitura e escrita é um fator limitante na diferenciação entre notícias falsas e verdadeiras, o que torna esses indivíduos tanto vítimas como propagadores ${ }^{15,16}$.

Apesar de apenas 31,1\% dos idosos brasileiros possuírem acesso à internet, houve um aumento expressivo do uso dessa tecnologia, principalmente, por meio do celular, que é utilizado por $65 \%$ dessa população ${ }^{6,17}$. Entretanto, os idosos ainda podem ser considerados digitalmente invisibilizados, visto que há a persistência da ideia de que eles estão distantes da internet e de mídias sociais. Por conta disso, ignora-se o ensino adequado para o uso devido dessas ferramentas ao grupo, o que os fragiliza perante as notícias claramente falsas ou incorretas. As ferramentas tecnológicas e seus usuários têm excluído o envelhecimento da população, e considera-se que os idosos não têm vontade e habilidades necessárias para fazer uso de todas as funções da tecnologia ${ }^{18}$.

Assim, combater a desinformação nas redes se torna necessário, e, para tanto, já há providências como a inciativa da OMS de compartilhar notícias por meio de redes sociais populares como Whatsapp, Facebook Messenger e Apple Business Chat, além do próprio MS que, de forma inovadora, disponibilizou um número de WhatsApp para receber informações virais, que são apuradas pelas áreas técnicas e respondidas oficialmente quanto à veracidade ${ }^{19}$.

Com a presente ação, os estudantes, de forma ativa, incentivaram a educação e o empoderamento dos idosos para que possam reconhecer notícias falsas e ter consciência da responsabilidade de compartilhar tais informações. Apontaram-se alguns passos para a identificação de fake news: verificar se há fonte, como indicação de site e autor do conteúdo; observar atentamente a data de publicação; fazer uma leitura além do título e subtítulo; pesquisar outros sites de conteúdo; avaliar se não se trata de uma piada ou brincadeira; compartilhar apenas após checagem da veracidade; e utilizar como ferramenta o canal "Saúde sem fake news" do MS, e, de acordo com cada realidade, os teleatendimentos municipais e nacionais ${ }^{20}$. Além disso, explicou-se sobre a importância da adoção de medidas preventivas e de combate à Covid-19, a forma correta de utilização de máscaras e a diminuição do contato entre pessoas, a fim de frear a propagação do vírus.

Percebe-se que o papel e a responsabilidade do profissional da saúde têm sido discutidos pela sua capacidade de conter a propagação dessas notícias por meio da promoção de fontes apropriadas e do esclarecimento das informações enganosas que são espalhadas em mídias sociais $^{21-24}$. É importante valorizar o papel do estudante de 
Medicina nesse sentido, que pode ser personagem-chave para discussões acerca de educação médica durante a pandemia, e no protagonismo ante o combate de fake news ${ }^{25-27}$. As funções do estudante durante esse período vêm sido muito debatidas, como o telemonitoramento e a ação direta em hospitais, clínicas e UBS, entretanto a presente ação também pode se tornar uma maneira de manter o acadêmico ativo, principalmente aqueles pertencentes ao grupo de risco.

Ainda em alusão à infodemia e à vulnerabilidade dos idosos, é importante considerar o impacto psíquico gerado pelo montante de notícias que pode causar sobrecarga emocional e mental dos indivíduos a elas sujeitos, deixando-os ansiosos e deprimidos $^{13}$. O fato de o indivíduo tornar-se ativo no processo de envelhecimento e manter-se dinâmico na sociedade está intrinsecamente relacionado com a socialização proveniente da inclusão tecnológica, a qual pode apresentar um panorama que remete a situações de incompreensão para o público idoso e causar sentimentos de discriminação, isolamento e ansiedade ${ }^{28}$. Somado a essa insegurança contemporânea pela dificuldade da habilidade de comunicação e interação, o contexto da pandemia contribui para a diminuir a autoestima dos idosos e aumentar o sentimento de distância. Buscou-se, assim, incentivar o bem-estar e as formas de relaxamento com o auxílio de atividades físicas aos idosos durante o isolamento social, de modo que seja promovido o processo de envelhecimento ativo e saudável.

No que se refere ao acesso e conhecimento dos acadêmicos de Medicina em relação à saúde do idoso, ainda há certa precariedade, haja vista a baixa implementação curricular de disciplinas obrigatórias ou optativas em geriatria e saúde do idoso ou envelhecimento ${ }^{29}$, fato contraditório em face da inversão da pirâmide etária brasileira7. Em 2010, revelou-se que, dentre 167 instituições médicas brasileiras pesquisadas, apenas $42 \%$ incluíam geriatria e outras disciplinas relacionadas ao envelhecimento em seus currículos $^{30}$. Seis anos depois, outro estudo demonstrou que, de 180 instituições de ensino superior da área médica, apenas $36 \%$ apresentavam disciplinas essenciais e eletivas no campo da geriatria, que as regiões com maior número de cursos de Medicina com essas disciplinas foram Sudeste e Sul ${ }^{29}$.

Outrossim, a ação relatada reitera importantes informações do Plano de Ação Internacional de Madri sobre Envelhecimento Humano, que traz como limitação ao acesso em saúde a falta de acessibilidade de serviços para a população geriátrica, com enfoque estratégico em países subdesenvolvidos, como o Brasil. Com isso em mente, o entendimento do acadêmico sobre a vivência do cotidiano dos idosos é uma possibilidade de ampliar o aprendizado sobre geriatria e gerontologia ${ }^{31,32}$. Assim, quando se implementa o contato entre idosos e acadêmicos, ocorre difusão de conhecimento geriátrico durante a graduação de Medicina, desde os períodos iniciais até os mais avançados. Ainda, possibilita um conhecimento maior acerca das principais demandas sobre a alfabetização em saúde e o período de pandemia do novo coronavírus.

\section{CONCLUSÃO}

Considerando o número total de idosos participantes, o caráter das perguntas respondidas e a suscetibilidade dessa população a informações equivocadas, a criação de um canal de comunicação com informações confiáveis, de dados verídicos e protocolos do MS e da OMS resultou em uma maior conscientização da população-alvo sobre a pandemia do novo coronavírus.

Faz-se necessário instruir os idosos sobre o fenômeno das fake news e buscar minimizar o compartilhamento por meios simples e práticos de verificação das informações. Mais além, a utilização de recursos de maior absorção, como áudios e vídeos, mostrou-se imprescindível para construir uma comunicação clara e instrutiva nos grupos. Ademais, a abordagem por meio de mídias digitais também proporcionou aos estudantes de Medicina uma maior compreensão sobre as vulnerabilidades e demandas da população geriátrica em tempos de crise, de forma a permitir maior contato e conhecimentos do cotidiano da gerontologia durante $o$ período de isolamento social.

Por fim, a partir da experiência vivenciada, tem-se como perspectiva futura a reflexão sobre a formação de um ambiente digital com informações seguras e confiáveis em saúde para a população idosa, baseado em evidências, bem como ações mais acessíveis, inclusivas e voltadas a esse público que vem crescendo dentro da internet, levando em consideração suas limitações, de modo que o acesso às informações dentro desses meios contribua para um processo de envelhecimento ativo e saudável.

\section{CONTRIBUIÇÃO DAS AUTORAS}

Angela Theresa Zuffo Yabrude, Andressa Caroline Martins de Souza, Catarine Wiggers de Campos e Loyse Bohn participaram da elaboração do estudo, da investigação de dados, do levantamento bibliográfico e da redação do artigo. Marcela Tiboni orientou todas as etapas e participou da revisão final e da edição do artigo.

\section{CONFLITO DE INTERESSES}

As autoras declaram não haver conflito de interesses neste estudo. 


\section{REFERÊNCIAS}

1. Pennycook G, McPhetres J, Zhang Y, Lu JG, Rand DG. Fighting COVID-19 misinformation on social media: experimental evidence for a scalable accuracy-nudge intervention. Psychological Science. 2020;31(7):770-80.

2. Orso D, Federici N, Copetti R, Vetrugno L, Bove T. Infodemic and the spread of fake news in the COVID-19-era. Eur J Emerg Med. 2020;27(5):3278. doi: 10.1097/MEJ.0000000000000713.

3. Vasconcellos-Silva PR, Castiel LD. COVID-19, as fake news e o sono da razão comunicativa gerando monstros: a narrativa dos riscos e os riscos das narrativas. Cad Saude Publica. 2020;395(10225):1-12.

4. Zarocostas J. How to fight an infodemic. The Lancet, 2020;395(10225):676.

5. Ofcom. Covid-19 news and information: consumption and attitudes. Results from week one of Ofcom's online survey [acesso em 11 ago 2020]. Disponível em: https://www.ofcom.org.uk/.

6. Instituto Brasileiro de Geografia e Estatística. Pesquisa Nacional por Amostra de Domicílios Contínua 2016-2017. Rio de Janeiro: IBGE; 2018 [acesso em 11 ago 2020]. Disponível em: https://biblioteca.ibge.gov.br/.

7. Instituto Brasileiro de Geografia e Estatística. Caminhos para uma melhor idade. Retratos: a Revista do IBGE. 2019:19-26 [acesso em 11 ago 2020]. Disponível em: https://biblioteca.ibge.gov.br/.

8. Instituto Brasileiro de Geografia e Estatística. Pesquisa Nacional por Amostra de Domicílios Contínua 2017-2018. Rio de Janeiro: IBGE; 2020 [acesso em 11 ago 2020]. Disponível em: https://biblioteca.ibge.gov.br/.

9. Costa FA, Silva AS, de Oliveira CBS, da Costa LCS, Paixão MES, Celestino MNS, et al. Covid-19: seus impactos clínicos e psicológicos na população idosa. Brazilian Journal of Development. 2020;6(7):49811-24.

10. Allcott $\mathrm{H}$, Gentzkow M. Social media and fake news in the 2016 election. $J$ Econ Perspect. 2017;31(2):211-36.

11. Manso MEG, Pinto IB, Hluchan VK, Oshiro LVS. Fake news e saúde da pessoa idosa. Rev. Longeviver. 2019;1(2):19-25.

12. Antunes MDL, Sanches T, Lopes C. A literacia da informação no combate às fake news: desafios e estratégias formativas no ensino superior. IX Encuentro Ibérico ECICIC; 2019. Barcelona: Universidad de Barcelona, 2019. [acesso em 11 ago 2020]. Disponível em: http://hdl.handle. net/10400.21/10323.

13. Organização Pan-Americana da Saúde. Entendendo a infodemia e a desinformação na luta contra a COVID-19, 2020 [acesso em 11 ago 2020]. Disponível em: https://iris.paho.org/.

14. Guess A, Nagler J, Tucker J. Less than you think: pevalence and predictors of fake news dissemination on Facebook. Sci Adv. 2019;5:1-8.

15. Ação Educativa. Indicador de Analfabetismo Funcional (INAF): INAF BRASIL 2018 [acesso em 11 ago 2020]. Disponível em: https:// acaoeducativa.org.br/.

16. Lima AL, Huaira-Contreras CA. Evolução do anafalbetismo funcional no Brasil: 2001-2010. Est. Aval. Educ. 2012;23(52):106-28

17. Centro Regional de Estudos para o Desenvolvimento da Sociedade da Informação. Pesquisa sobre o Uso das Tecnologias de Informação e Comunicação nos Domicílios Brasileiros - TIC Domicílios 2019 [acesso em 11 ago 2020]. Disponível em: CGI.br/NIC.br.
18. Raymundo TM. Aceitação de tecnologia por idoso [dissertação]. São Carlos: Universidade de São Paulo; 2013 [acesso em 11 ago 2020]. Disponível em: https://teses.usp.br/.

19. Naeem SB, Bhatti R. The Covid-19 "infodemic": a new front for information professionals. Health Info Libr J. 2020 [acesso em 9 ago 2020]. Disponível em: https://onlinelibrary.wiley.com/doi/full/10.1111/hir.12311?af=R.

20. Swire-Thompson B, Lazer D. Public health and online misinformation: challenges and recommendations. Annu Rev Public Health. 2020; 41(1): 433-51

21. Chou W-YS, Oh A, Klein WMP. Addressing health-related misinformation on social media. JAMA. 2018;320(23):2417-8.

22. Bode L, Vraga EK. See something, say something: correction of global health misinformation on social media. Health Commun. 2018;33:1131-40.

23. Erku DA, Belachew SA, Abrha S, Sinnollareddy M, Thomas J, Steadman KJ, et al. When fear and misinformation go viral: pharmacists' role in deterring medication misinformation during the "infodemic" surrounding COVID-19. Res Social Adm Pharm. 2020:1-10.

24. Shimizu K. 2019-nCoV, fake news, and racism. The Lancet. 2020; 395(10225): 685-6.

25. Meganne N, Ferrel JJR. The impact of COVID-19 on medical education. Cureus. 2020;12(3):e7492 [acesso em 10 ago 2020]. Disponível em: https://pubmed.ncbi.nlm.nih.gov/32368424/.

26. Elsevier. Medical students tackle coronavirus misinformation. 2019 [acesso em 11 ago. 2020]. Disponível em: https://www.elsevier.com/ connect/medical-students-tackle-coronavirus-misinformation.

27. Segaren S. Medical students at Harvard fight COVID-19 fake news, one tweet at a time. Study International 2020 [acesso em 11 de agosto de 2020]. Disponível em: https://www.studyinternational.com/news/harvardstudents-flatten-curve/.

28. Sousa ACSN, Lodovici FMM, Silveira NDR, Arantes RPG. Alguns apontamentos sobre o idadismo: a posição de pessoas idosas diante desse agravo à sua subjetividade. Estud Interdiscip Envelhec. 2014;19(3):853-77.

29. Cunha ACNP, Cunha NNP, Barbosa MT. Geriatric teaching in Brazilian medical schools in 2013 and considerations regarding adjustment to demographic and epidemiological transition. Rev Assoc Med Bras. 2016;62(2):179-83.

30. Pereira AMVB, Feliz MC, Schawanke CHA. Ensino de geriatria nas faculdades de Medicina brasileiras. Rev Geriatr Gerontol. 2010;4(4):179-85.

31. United Nations. The Second World Assembly on Ageing 2002. The third meeting of the technical commmitee for the Second World Assembly on Ageing; 2002 [aceso em 5 ago 2020]. Disponível em: http://undesadspd. org/Portals/o/ageing/documents/Fulltext-E.pdf.

32. Brasil VJW, Batista NA. O ensino de geriatria e gerontologia na graduação médica. Rev Bras Educ Med. 2015;39(3):344-51. 